Article

\title{
The Drosophila melanogaster Metabolic Response against Parasitic Nematode Infection Is Mediated by TGF- $\beta$ Signaling
}

\author{
Yaprak Ozakman, Trishya Pagadala, Dhaivat Raval and Ioannis Eleftherianos * \\ Infection and Innate Immunity Laboratory, Department of Biological Sciences, Institute for Biomedical Sciences, \\ The George Washington University, Washington, DC 20052, USA; yozakman@gwmail.gwu.edu (Y.O.); \\ trishya.pagadala@gmail.com (T.P.); draval7@gwmail.gwu.edu (D.R.) \\ * Correspondence: ioannise@gwu.edu; Tel.: +1-202-994-2367
}

Received: 25 May 2020; Accepted: 28 June 2020; Published: 29 June 2020

check for updates

\begin{abstract}
The nematode Heterorhabditis bacteriophora, its mutualistic bacterium Photorhabdus luminescens, and the fruit fly Drosophila melanogaster establish a unique system to study the basis of infection in relation to host metabolism. Our previous results indicate that the Transforming Growth Factor $\beta$ (TGF- $\beta$ ) signaling pathway participates in the D. melanogaster metabolic response against nematode parasitism. However, our understanding of whether the presence of Photorhabdus bacteria in Heterorhabditis nematodes affects the metabolic state of $D$. melanogaster during infection is limited. Here, we investigated the involvement of TGF- $\beta$ signaling branches, Activin and Bone Morphogenetic Protein (BMP), in the D. melanogaster metabolic response against axenic (lacking bacteria) or symbiotic (containing bacteria) H. bacteriophora infection. We show that BMP signaling mediates lipid metabolism against axenic or symbiotic $H$. bacteriophora and alters the size of fat body lipid droplets against symbiotic nematode infection. Also, following symbiotic $H$. bacteriophora infection, Activin signaling modulates sugar metabolism. Our results indicate that Activin and BMP signaling interact with the D. melanogaster metabolic response to H. bacteriophora infection regardless of the presence or absence of Photorhabdus. These findings provide evidence for the role of TGF- $\beta$ signaling in host metabolism, which could lead to the development of novel treatments for parasitic diseases.
\end{abstract}

Keywords: D. melanogaster; Heterorhabditis; metabolism; parasitism; TGF- $\beta$ signaling

\section{Introduction}

The innate immune response against microbial infection is often associated with the metabolic state of the host [1-6]. In turn, the metabolic state of an organism is a critical determinant of a functional immune system. Several components of the immune system modulate metabolism, and together these processes are paramount for tissue and organismal homeostasis [7].

The nematode parasite Heterorhabditis bacteriophora together with its symbiotic bacteria Photorhabdus luminescens and the fruit fly Drosophila melanogaster constitute a well-suited model to dissect the molecular basis of infection in relation to the host metabolic response. Heterorhabditis nematodes infect various insects at their infective juvenile (IJ) stage. Upon entry, the nematodes expel their symbiotic bacteria into the insect hemolymph, where the bacteria secrete toxins and virulence factors to suppress the insect immune system [8,9]. The bacteria also serve as an essential nutrient source required for nematode growth and proliferation. When nematode numbers reach high density and resources are depleted, the IJs which are colonized by the bacteria, emerge from the insect cadaver to search for a new host [10]. 
In addition to its pivotal role in several developmental processes such as axis formation, body patterning, and morphogenesis, the Transforming Growth Factor- $\beta$ (TGF- $\beta$ ) signaling pathway modulates anti-nematode immunity in D. melanogaster [6,11-15]. Components of the TGF- $\beta$ signaling pathway are expressed in immunologically, and metabolically active tissues in both vertebrates and invertebrates, and their roles in regulating immunity and metabolism have been gaining attention [16,17]. Similar to vertebrates, the TGF- $§$ pathway in $D$. melanogaster is composed of two signaling branches named Activin and Bone Morphogenetic Protein (BMP). The TGF- $\$$ signaling pathway is initiated by the binding of an extracellular ligand to a transmembrane receptor complex of serine/threonine kinases [16,18]. The Activin branch is activated by Activin- $\beta$ (Act $\beta$ ), Dawdle (Daw) and Myoglianin (Myo) ligands, and the BMP branch is activated by Decapentaplegic (Dpp), Glass-bottom boat (Gbb) and Screw (Scw) [19]. Activation of the receptor leads to phosphorylation of downstream transcription factors that regulate the expression of target genes [20].

Previous studies have shown that extracellular ligands Daw and Dpp are involved in the immune response of D. melanogaster adult flies against parasitic nematode infection [14,15]. In addition, recent work has demonstrated that the TGF- $\$$ signaling Activin and BMP branches participate in the $D$. melanogaster metabolic response against the nematode parasite $H$. gerrardi [6]. Both signaling branches regulate lipid metabolism, and the Activin pathway only interferes with glycogen metabolism upon $H$. gerrardi infection. However, our understanding of whether Heterorhabditis nematodes are capable of altering the metabolic state of D. melanogaster without any contribution from their associated bacterial symbionts remains currently unknown. Therefore, it is essential to separate the two symbiotic partners, nematodes and bacteria, and study the metabolic capacity of the insect host to infection with axenic (lacking Photorhabdus bacteria) or symbiotic (containing Photorhabdus bacteria) nematodes independently.

In order to elucidate the role and regulation of TGF- $\beta$ signaling branches in the D. melanogaster metabolic response against infection with axenic or symbiotic $H$. bacteriophora nematodes, we used D. melanogaster larvae carrying loss-of-function mutations in daw and $d p p$ genes coding for extracellular ligands in the Activin and BMP signaling branches, respectively. We measured carbohydrate, lipid and ATP levels, and determined the size of lipid droplets in the fat body of these mutants upon infection with axenic or symbiotic nematodes. Results obtained from this study provide novel insight into the role of TGF- $\$$ signaling in host metabolism in the context of nematode infection and thus may lead to the identification of new strategies for treating parasitic infectious diseases.

\section{Materials and Methods}

\subsection{Fly Stocks}

Fly stocks were maintained on D. melanogaster medium (Meidi Laboratories) supplemented with baker's yeast (Carolina Biological Supply, Burlington, NC, USA) at $25^{\circ} \mathrm{C}$, and a 12:12-h light:dark photoperiodic cycle. Flies carrying a spontaneous dpp ${ }^{s 1}$ mutation (strain 397, Bloomington, IL, USA) and a P-bac insertion Pbac $\{$ XP\}daw05680 (strain d05680, Exelixis, Boston, MA, USA) were used in this study, as previously described [6]. Fly line $w^{1118}$ (strain 3605, Bloomington, IL, USA) was used as background control. Late second to early third instar larvae were used for all experiments.

\subsection{Nematode Stocks}

Infective juveniles (IJs) of $H$. bacteriophora strain TT01 were amplified through the fifth to sixth instar larvae of the wax moth Galleria mellonella using the water trap technique [21]. Axenic H. bacteriophora nematodes lacking their symbiotic P. luminescens bacteria were generated by a previously established technique [22]. Prior to use, axenic nematodes were surface-sterilized in $10 \%$ bleach solution and rinsed five times with water to remove the bleach residue. All nematodes were used within one to four weeks after collection. 


\subsection{Larval Infection}

D. melanogaster larvae were infected with axenic or symbiotic $H$. bacteriophora IJs in microtiter 96-well plates, containing $100 \mu \mathrm{L}$ of $1.25 \%$ agarose in each well. The suspension of IJs (100 nematodes in $10 \mu \mathrm{L}$ of sterile distilled water) was added into each well of the microtiter plate followed by a transfer of a single larva. Sterile distilled water $(10 \mu \mathrm{L})$ was used as the uninfected control. The plate was covered with a sealing film (USA Scientific, Ocala, FL, USA) and air holes were pierced for aeration. Plates were kept in dark at room temperature for $24 \mathrm{~h}$. At the 24-h time point, infected and uninfected larvae, were collected and frozen at $-80^{\circ} \mathrm{C}$, or immediately used in experiments. Each infection experiment was performed three times with biological duplicates that included larvae from different generations.

\subsection{RNA Analysis}

Extraction of total RNA from five to seven D. melanogaster larvae was performed using TRIzol ${ }^{\mathrm{TM}}$ reagent following the manufacturer's instructions. Reverse transcription and Quantitative RT-PCR (qRT-PCR) experiments were carried as previously described using gene specific primers for Ribosomal protein L32 (RpL32), Drosophila insulin-like peptide 6 (Dilp6), Drosophila insulin-like peptide 3 (Dilp3), and forkhead box-O (fOXO) (Table 1) [6]. Each experiment was run in biological duplicates and repeated three times.

Table 1. Primers and their sequences used in quantitative RT-PCR experiments.

\begin{tabular}{ccccc}
\hline Gene & Accession No & Primer $\left(5^{\prime}-\mathbf{3}^{\prime}\right)$ & Sequence & Tm $\left({ }^{\circ} \mathbf{C}\right)$ \\
\hline $\boldsymbol{R} p$ L32 & CG7939 & $\begin{array}{c}\text { Forward } \\
\text { Reverse }\end{array}$ & $\begin{array}{c}\text { GATGACCATCCGCCCAGCA } \\
\text { CGGACCGACAGCTGCTTGGC }\end{array}$ & 60 \\
\hline Dilp6 & CG14049 & $\begin{array}{c}\text { Forward } \\
\text { Reverse }\end{array}$ & $\begin{array}{c}\text { ATATGCGTAAGCGGAACGGT } \\
\text { GCAAGAGCTCCCTGTAGGTG }\end{array}$ & 57 \\
\hline Dilp3 & CG14167 & $\begin{array}{c}\text { Forward } \\
\text { Reverse }\end{array}$ & $\begin{array}{c}\text { AGAGAACTTTGGACCCCGTGAA } \\
\text { TGAACCGAACTATCACTCAACAGTCT }\end{array}$ & 59 \\
\hline fOXO & CG3143 & $\begin{array}{c}\text { Forward } \\
\text { Reverse }\end{array}$ & $\begin{array}{c}\text { AGGCGCAGCCGATAGACGAATTTA } \\
\text { TGCTGTTGACCAGGTTCGTGTTGA }\end{array}$ & 60 \\
\hline
\end{tabular}

\subsection{Measurement of Triglyceride, Trehalose, Glucose, and Glycogen Levels}

D. melanogaster larvae were infected with axenic or symbiotic H. bacteriophora IJs and $24 \mathrm{~h}$ following infection, seven to nine larvae were collected and rinsed in $1 \times$ PBS. Larvae were then homogenized with a pellet pestle on ice in $100 \mu \mathrm{L}$ of $1 \times$ PBS to determine glucose and glycogen levels, or in $100 \mu \mathrm{L}$ Trehalase buffer (TB; $5 \mathrm{mM}$ Tris $\mathrm{pH}$ 6.6, $137 \mathrm{mM} \mathrm{NaCl}, 2.7 \mathrm{mM} \mathrm{KCl}$ ) to assess trehalose levels, or in $100 \mu \mathrm{L}$ of PBST (1× PBS $+0.05 \%$ Tween 20$)$ to measure triglyceride levels, using previously established protocols [23]. Protein quantification was determined by Pierce ${ }^{\mathrm{TM}}$ BCA Protein Assay Kit (Thermo Fisher Scientific; 23227, Waltham, MA, USA).

Triglyceride levels were determined by adding diluted samples (1:1 in PBS-Tween) and $200 \mu \mathrm{L}$ of Infinity ${ }^{\mathrm{TM}}$ Triglycerides Liquid Stable Reagent (Thermo Fisher Scientific; TR22421) into a clear 96-well plate, which was incubated for $30 \mathrm{~min}$ at $37^{\circ} \mathrm{C}$. Following the incubation, absorbance was measured at $540 \mathrm{~nm}$. The glycerol standard curve was used to calculate triglyceride concentrations.

To determine the levels of trehalose, samples were initially diluted 1:3 in TB and further diluted 1:1 in either TB or Trehalase Stock (TS; $3 \mu \mathrm{L}$ of porcine trehalase in $1 \mathrm{~mL}$ of TB). Following an incubation at $37^{\circ} \mathrm{C}$ for $24 \mathrm{~h}$ in a clear 96-well plate, hexokinase (Glucose Assay Reagent, Sigma-Aldrich; G3293, St. Louis, MO, USA) reagent $(100 \mu \mathrm{L})$ was added to each well and the absorbance was measured at $340 \mathrm{~nm}$. Trehalose levels were calculated from samples digested in TS by subtracting the amount of free glucose.

The levels of glucose and glycogen were measured by initially diluting the samples in 1:3 in PBS and then further diluting them to 1:1 in either amyloglucosidase stock solution $(1.5 \mu \mathrm{L}$ of 
amyloglucosidase in $1 \mathrm{~mL}$ of PBS, Sigma-Aldrich) or PBS. Following the incubation of diluted samples $(30 \mu \mathrm{L})$ at $37^{\circ} \mathrm{C}$ for $60 \mathrm{~min}$ in a clear 96-well plate, hexokinase reagent $(100 \mu \mathrm{L})$ was added to each well and samples were incubated at room temperature for an additional $15 \mathrm{~min}$. Absorbance was measured at $340 \mathrm{~nm}$. Glucose standard curve was used to determine glucose levels. Glycogen levels were calculated by subtracting the absorbance of glucose from the absorbance of samples diluted with amyloglucosidase stock.

The amounts of triglycerides, trehalose, glucose, and glycogen were calculated relative to the amount of proteins in each sample. All experiments were run in biological duplicates and repeated three times.

\subsection{Measurement of Cholesterol and ATP Levels}

D. melanogaster larvae were infected with axenic or symbiotic $H$. bacteriophora IJs and at $24 \mathrm{~h}$ post-infection, seven to nine larvae were collected and homogenized either in $100 \mu \mathrm{L}$ of $1 \times$ reaction buffer provided by Amplex Red Cholesterol Assay Kit (Invitrogen; A12216, Carlsbad, CA, USA) for cholesterol quantification, or in ATP reaction mix (Molecular Probes ATP kit; A22066, Eugene, OR, USA) for measuring ATP levels. The amount of proteins was determined by Pierce ${ }^{\mathrm{TM}}$ BCA Protein Assay Kit (Thermo Fisher Scientific; 23227).

To determine cholesterol levels, $50 \mu \mathrm{L}$ of samples were mixed with $90 \mu \mathrm{L}$ of the reaction mix provided by the kit in black 96-well plates (Fisher Scientific; 509051574) and incubated for 30 min at $37^{\circ} \mathrm{C}$. Fluorescence was measured with excitation at $530 \mathrm{~nm}$ and emission at $590 \mathrm{~nm}$.

To measure ATP levels, samples were initially diluted 1:10 and subsequently diluted further 1:75 in dilution buffer ( $25 \mathrm{mM}$ of Tris, $100 \mu \mathrm{M}$ of EDTA). Samples were then transferred to individual wells of a 96-well plate and the luciferase reaction mix $(100 \mu \mathrm{L})$ was added to each well. Luminescence was measured immediately. Each experiment was performed in biological duplicates and repeated three times.

\subsection{Lipid Droplet Staining}

At $24 \mathrm{~h}$ following infection of D. melanogaster larvae with axenic or symbiotic H. bacteriophora, fat body tissues of $10 \mathrm{D}$. melanogaster larvae were dissected and fixed in $4 \%$ paraformaldehyde prepared in PBS at room temperature for $30 \mathrm{~min}$. Tissues were then rinsed with PBS and incubated in the dark for $30 \mathrm{~min}$ in $0.05 \%$ of Nile red diluted 1:1000 in $1 \mathrm{mg} / \mathrm{mL}$ of methanol. ProLong ${ }^{\mathrm{TM}}$ Diamond AntiFade Mountant with DAPI (Life Technologies; P36962, Carlsbad, CA, USA) was used to mount tissues. Lipid droplets were visualized using a Zeiss LSM 510 confocal microscope. Quantification of lipid droplet size was assessed by measuring the area of the four largest lipid droplets per cell from four fat body cells using ImageJ software (National Institutes of Health, Bethesda, MD, USA).

\subsection{Statistical Analysis}

GraphPad Prism7 was used for data plotting and statistical analyses. Statistical analyses of all experimental results were performed using one-way analysis of variance (ANOVA).

\section{Results}

\subsection{D. melanogaster daw Mutant Larvae Contain Lower Levels of Trehalose Upon Symbiotic H. bacteriophora} Infection

Sugar metabolism is essential for maintenance of cellular energy levels in insects. D. melanogaster contains glucose and trehalose as circulating sugars, and glycogen as stored sugar [24]. Recent work in D. melanogaster larvae has revealed a link between regulation of glycogen metabolism and Activin signaling in response to infection with $H$. gerrardi parasitic nematodes. Specifically, D. melanogaster daw mutant larvae infected with $H$. gerrardi nematodes contain elevated glycogen levels compared to uninfected daw mutant larvae [6]. To investigate whether the Activin and BMP signaling branches 
alter sugar metabolism in D. melanogaster in response to H. bacteriophora axenic or symbiotic nematodes, we estimated trehalose, glucose, and glycogen levels $24 \mathrm{~h}$ post-infection. We found that daw mutant larvae contained significantly higher levels of trehalose in the absence of infection $(p<0.0001)$ and in response to infection with axenic nematodes $(p<0.0001)$ compared to their background controls $\left(w^{1118}\right)$ (Figure 1A). However, levels of trehalose were significantly lower in daw mutant larvae upon infection with symbiotic $H$. bacteriophora compared to uninfected daw mutants $(p=0.0004)$ and larvae infected with axenic nematodes $(p=0.0002)$. In addition, $d p p$ mutant larvae infected with symbiotic nematodes contained significantly increased amounts of trehalose compared to those infected with axenic nematodes $(p=0.0272)$. In contrast, daw mutant larvae had lower levels of glucose in the absence of infection $(p=0.0017)$ and in response to axenic $(p=0.0386)$ or symbiotic H. bacteriophora challenge $(p=0.0036)$ than their background controls $\left(w^{1118}\right)$ (Figure $\left.1 \mathrm{~B}\right)$. We did not observe any statistically significant changes in glycogen levels between daw and $d p p$ mutant larvae infected with axenic or symbiotic nematodes relative to uninfected controls (Figure $1 \mathrm{C}$ ). These results indicate that the Activin branch of TGF- $\beta$ signaling in D. melanogaster modulates metabolism of circulating sugars in the context of a nematode challenge by increasing the levels of trehalose in larvae responding to symbiotic $H$. bacteriophora infection.

A.

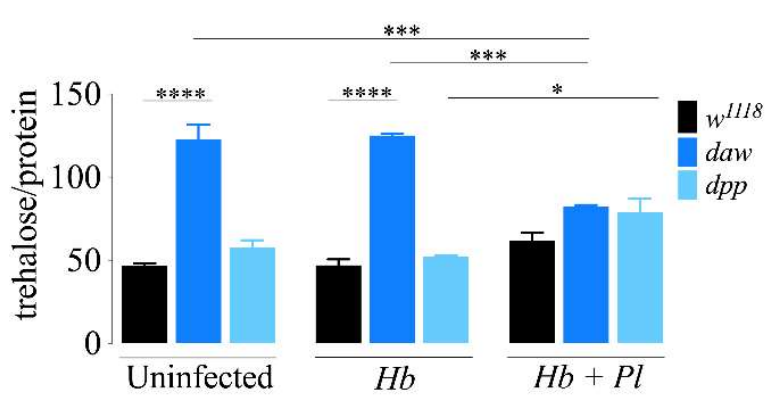

B.

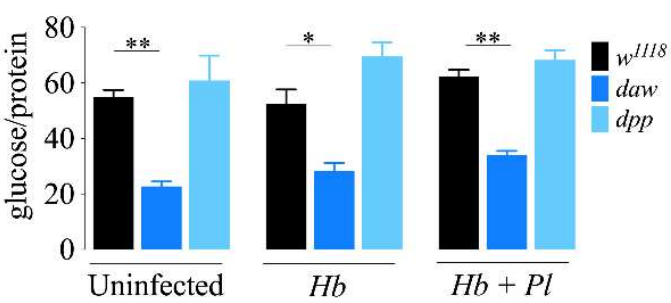

c. Glycogen

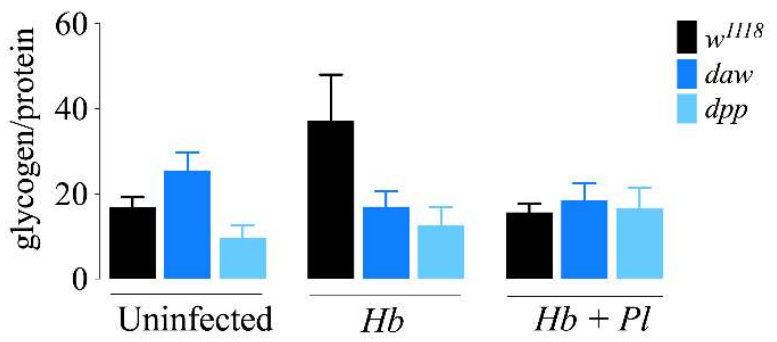

Figure 1. Carbohydrate metabolism in Drosophila melanogaster TGF-ß mutant larvae $24 \mathrm{~h}$ after infection with axenic (Heterorhabditis bacteriophora, $\mathrm{Hb}$ ) or symbiotic (Heterorhabditis bacteriophora + Photorhabdus luminescens, $\mathrm{Hb}+\mathrm{Pl}$ ) nematodes. (A) Levels of trehalose in daw and dpp mutant larvae infected with parasitic nematodes. Trehalose levels are elevated in uninfected daw mutant larvae and in daw mutants infected with axenic $H$. bacteriophora nematodes compared to their respective background controls $\left(w^{1118}\right)\left({ }^{* * *} p<0.0001\right)$. In addition, trehalose levels are decreased in daw mutant larvae infected with symbiotic nematodes compared to the uninfected daw mutants $(* * *=0.0004)$ and daw mutants infected with axenic nematodes (*** $p=0.0002)$. Trehalose content in $d p p$ mutant larvae infected with $H$. bacteriophora symbiotic nematodes is significantly higher than in $d p p$ mutants infected with axenic 
nematodes (* ${ }^{*}=0.0272$ ). (B) Levels of glucose in infected daw and $d p p$ mutant larvae. Levels of glucose are reduced in uninfected daw mutant larvae $(* * p=0.0017)$ and those infected with either axenic (* $p=0.0386$ ) or symbiotic H. bacteriophora (** $p=0.0036)$ compared to their background controls $\left(w^{1118}\right)$. (C) Levels of glycogen in infected daw and $d p p$ mutant larvae. There is no significant change in the levels of glycogen in TGF- $\beta$ mutant larvae upon infection with axenic or symbiotic nematodes. The experiments were performed in biological duplicates and repeated three times $(\mathrm{N}=42-54)$.

3.2. D. melanogaster dpp Mutants Express dilp3 at Higher Levels in Response to Symbiotic H. bacteriophora Infection

The insulin signaling pathway controls essential processes, including the metabolic response in D. melanogaster [25]. Drosophila insulin-like peptides (Dilps) maintain the balance between stored and circulating carbohydrates, and regulate lipid metabolism [26,27]. Previous evidence indicates that lack of dilp3 results in elevated levels of trehalose and glycogen, and lack of dilp6 increases stored lipid levels in the absence of infection in D. melanogaster [28,29]. In addition, it has been shown that insulin signaling interacts with the TGF- $§$ signaling in the absence of infection. More precisely, the Activin pathway interacts with insulin signaling through Daw by positively regulating the release of Dilps in D. melanogaster larvae [30]. To investigate a potential link between insulin signaling and TGF- $\beta$ signaling in response to parasitic nematode infection, we used qRT-PCR and gene-specific primers to determine the transcript levels of dilp3, dilp6, and transcription factor fOXO in daw and $d p p$ mutant larvae $24 \mathrm{~h}$ after infection with $H$. bacteriophora axenic or symbiotic nematodes. We found no statistically significant differences in dilp3 and dilp6 levels in daw mutant larvae after infection with $H$. bacteriophora axenic or symbiotic nematodes compared to their background controls $\left(w^{1118}\right)$ (Figure 2A,B). However, expression of $f O X O$ was reduced in background control larvae $\left(w^{1118}\right)$ infected with $H$. bacteriophora symbiotic nematodes compared to uninfected controls (Figure 2C; $p=0.0098$ ). We further noticed that the expression of dilp3 was higher in $d p p$ mutant larvae infected with symbiotic $H$. bacteriophora compared to $d p p$ mutants infected with axenic nematodes (Figure $3 \mathrm{~A} ; p=0.0486$ ). We observed no statistically significant differences in the transcript levels of dilp6 in dpp mutant larvae infected with either axenic or symbiotic nematodes compared to their background controls $\left(w^{1118}\right)$ (Figure 3B). These results imply that the activity of BMP branch of the TGF- $\$$ signaling pathway in D. melanogaster larvae might be involved in suppressing dilp3 expression upon infection with $H$. bacteriophora symbiotic nematodes. 
A.

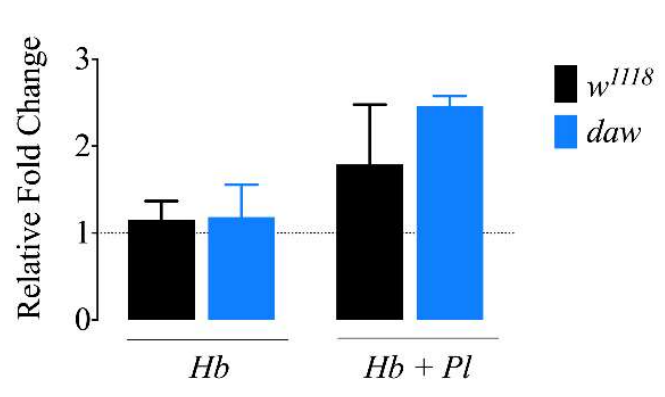

B.

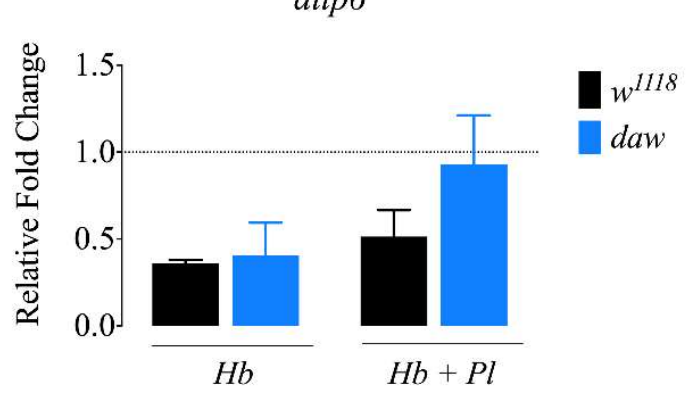

C.

fOXO

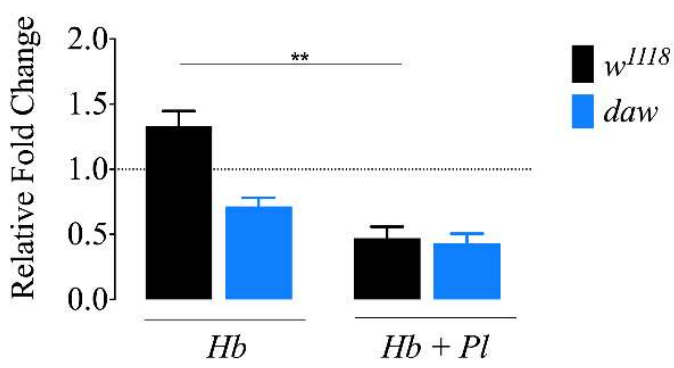

Figure 2. Expression of dilp3, dilp 6 and fOXO in Drosophila melanogaster daw mutant and background control larvae $24 \mathrm{~h}$ after infection with axenic (Heterorhabditis bacteriophora, $\mathrm{Hb}$ ) or symbiotic (Heterorhabditis bacteriophora + Photorhabdus luminescens, $\mathrm{Hb}+\mathrm{Pl}$ ) nematodes. $(\mathbf{A}, \mathbf{B})$ There is no significant change in the expression of dilp3 and dilp6 in daw mutant larvae after infection with H. bacteriophora axenic or symbiotic nematodes compared to their background control $\left(w^{1118}\right)$. (C) Expression of $f O X O$ is reduced in background control larvae $\left(w^{1118}\right)$ when infected with H. bacteriophora symbiotic nematodes compared to uninfected controls $\left({ }^{* *} p=0.0098\right)$. Dotted line at 1 indicates normalization of fold change relative to uninfected controls. The experiments were performed in biological duplicates and repeated three times $(N=30-42)$. 
A.

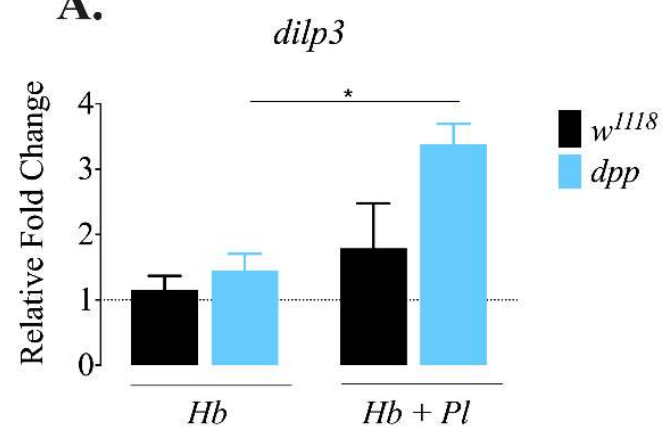

B.

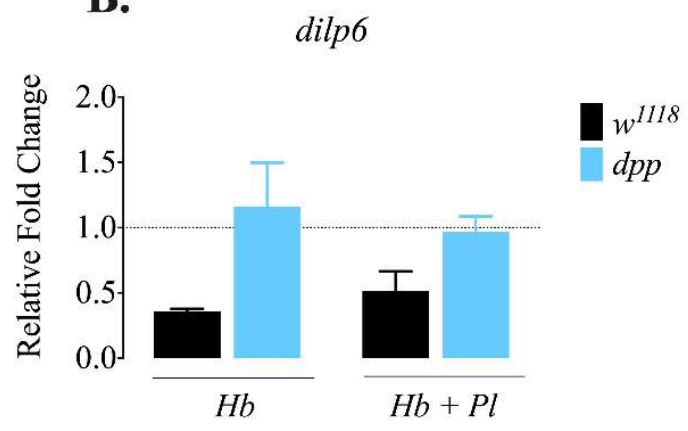

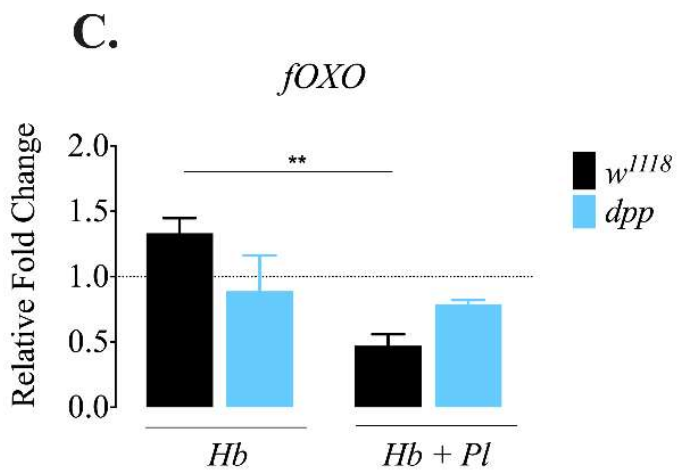

Figure 3. Expression of dilp3, dilp6 and fOXO in Drosophila melanogaster dpp mutant and background control larvae $24 \mathrm{~h}$ after infection with axenic (Heterorhabditis bacteriophora, $\mathrm{Hb}$ ) or symbiotic (Heterorhabditis bacteriophora + Photorhabdus luminescens, $\mathrm{Hb}+\mathrm{Pl}$ ) nematodes. (A) Expression of dilp3 increases in $d p p$ mutant larvae infected with symbiotic $H$. bacteriophora compared to dpp mutants infected with axenic H. bacteriophora ( $\left.{ }^{*} p=0.0486\right)$. (B) There is no significant change in the expression of dilp6 in $d p p$ mutant larvae infected with either axenic or symbiotic nematodes compared to their background control $\left(w^{1118}\right)$. (C) Expression of $f O X O$ is reduced in background control larvae $\left(w^{1118}\right)$ infected with symbiotic $H$. bacteriophora compared to background control larvae infected with axenic nematodes $(* * p=0.0098)$. Dotted line at 1 indicates normalization of fold change relative to uninfected controls. The experiments were performed in biological duplicates and repeated three times $(\mathrm{N}=$ 30-42).

3.3. The BMP Branch Regulates Metabolism of Stored Fats in D. melanogaster Larvae in the Context of H. bacteriophora Infection

Lipid metabolism has a central role in maintaining energy homeostasis in insects. In D. melanogaster, stored fats such as triacylglycerol (TAG) and cholesterol mostly accumulate in the fat body, the functional equivalent of the mammalian liver [31]. Previous work has demonstrated that in the absence of infection, TGF- $\beta$ signaling activity in hepatocytes contributes to lipid accumulation in mice [32]. In addition, the extracellular ligand Gbb in the BMP branch is required in the fat body of uninfected D. melanogaster larvae to modulate lipid metabolism [33]. To investigate the interaction between the Activin or the BMP branch of the TGF- $\beta$ signaling and the metabolism of stored lipids in the context of parasitic nematode infection, we determined the levels of TAG and free cholesterol in daw and $d p p$ loss-of-function mutant larvae $24 \mathrm{~h}$ after infection with axenic or symbiotic $H$. bacteriophora. We found that TAG levels in $d p p$ mutant larvae significantly decreased after infection with symbiotic $H$. bacteriophora compared to background control larvae $\left(w^{1118}\right)$ (Figure $\left.4 \mathrm{~A} ;{ }^{* * *} p=0.0004\right)$. On the contrary, cholesterol content was reduced in $d p p$ mutant larvae in response to infection with axenic nematodes compared to background controls (Figure 4B; $p=0.0038$ ). However, we found no statistically significant differences in TAG 
and cholesterol levels in daw mutant larvae after infection with $H$. bacteriophora axenic or symbiotic nematodes compared to their control individuals (Figure 4A,B).

A.

Triglycerides

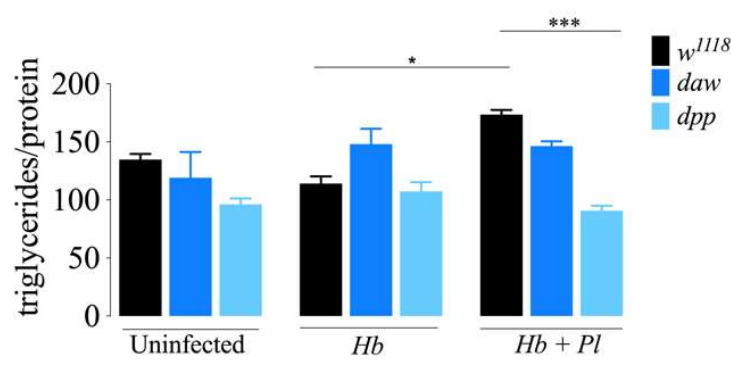

C.

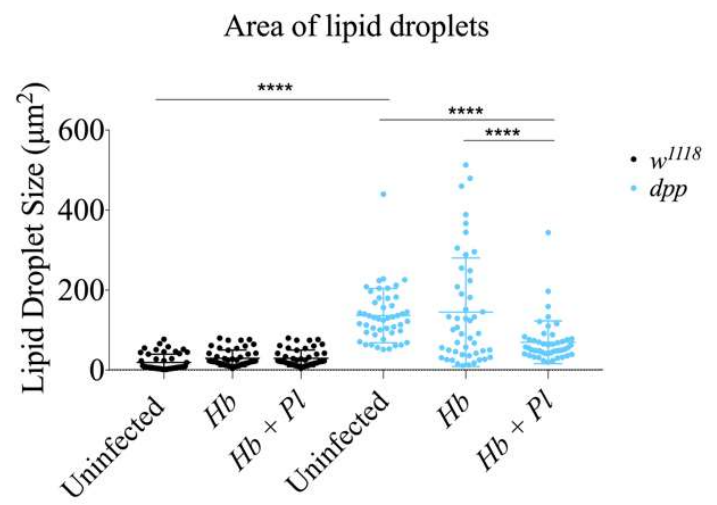

B.

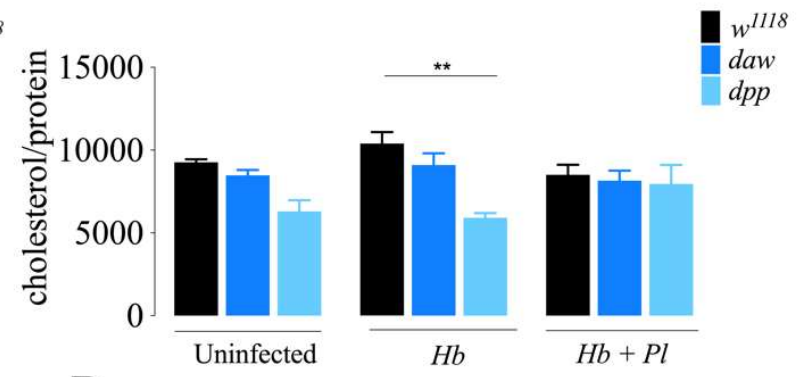

D.

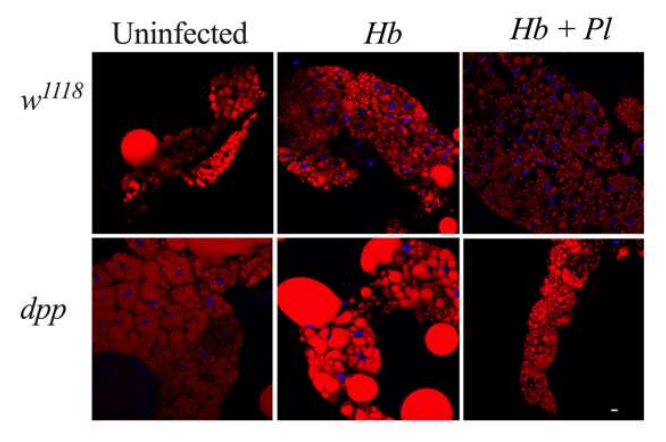

Figure 4. Lipid metabolism in Drosophila melanogaster TGF- $\beta$ mutant larvae $24 \mathrm{~h}$ after infection with axenic (Heterorhabditis bacteriophora, $\mathrm{Hb}$ ) or symbiotic (Heterorhabditis bacteriophora + Photorhabdus luminescens, $\mathrm{Hb}+\mathrm{Pl}$ ) nematodes. (A) Triglyceride levels in TGF- $\$$ mutant larvae infected with parasitic nematodes. Triglyceride content in $d p p$ mutant larvae significantly decreases after infection with $H$. bacteriophora symbiotic nematodes compared to background control $\left(w^{1118}\right)$ larvae $\left.{ }^{* * *} p=0.0004\right)$. In addition, following symbiotic $H$. bacteriophora infection, level of triglycerides increase in background control $\left(w^{1118}\right)$ larvae compared to larvae infected with axenic nematodes $\left({ }^{*} p=0.0164\right)$. (B) Cholesterol levels in TGF- $\$$ mutant larvae infected with parasitic nematodes. Cholesterol content is reduced in $d p p$ mutants infected with axenic $H$. bacteriophora compared to background control larvae $\left({ }^{* *} p=0.0038\right)$. (C) Size of lipid droplets in the fat body of D. melanogaster dpp mutant larvae $24 \mathrm{~h}$ after infection with $H$. bacteriophora axenic or symbiotic nematodes (**** $p<0.0001$ ). (D) Confocal microscopy images of lipid droplets stained with Nile Red (red) and DAPI (blue) in the fat body of H. bacteriophora-infected or uninfected $D$. melanogaster dpp mutant larvae and their background controls $\left(w^{1118}\right)$. Scale bar is 10 microns. Triglyceride and cholesterol quantification experiments were performed in biological duplicates and repeated three times $(N=42-54)$. Lipid droplet staining was performed by measuring the four largest lipid droplets per cell from four fat body cells.

Lipid droplets are specialized energy storage organelles that play an essential role in lipid metabolism and cellular homeostasis in D. melanogaster [34]. Recent work has shown that the size of lipid droplets in D. melanogaster dpp mutant larvae infected with $H$. gerrardi nematodes increases compared to uninfected larvae [6]. To further assess the interaction between the BMP branch and lipid metabolism, we stained fat body lipid droplets with Nile red and DAPI, and measured lipid droplet size in $d p p$ loss-of-function mutant larvae $24 \mathrm{~h}$ after infection with $H$. bacteriophora axenic or symbiotic nematodes (Figure 4C,D). We found that the size of fat body lipid droplets significantly decreased in $d p p$ mutant larvae after infection with $H$. bacteriophora symbiotic nematodes compared to uninfected larvae (Figure $4 C ; p<0.0001$ ). In addition, $d p p$ mutant larvae infected with symbiotic 
nematodes contained smaller lipid droplets than larvae infected with axenic nematodes $(p<0.0001)$. These findings suggest that the BMP/Dpp branch of the TGF- $\beta$ signaling pathway in D. melanogaster larvae regulates the metabolism of TAG in response to H. bacteriophora symbiotic nematodes, and cholesterol in response to axenic nematodes. It also modulates fat body lipid droplet size upon infection with symbiotic nematodes.

\subsection{ATP Levels in D. melanogaster daw and dpp Mutant Larvae Are Unaffected in Response to H. bacteriophora Infection}

Adenosine triphosphate (ATP) is found in all living cells and is responsible for energy storage and transfer. Quantification of ATP provides direct information on cellular energy levels which is associated with the metabolic state of the organism [23]. To understand whether the Activin and the BMP branches of the TGF- $\beta$ signaling pathway modulate cellular energy levels in D. melanogaster responding to nematode parasites containing or lacking their symbiotic bacteria, we infected larvae with $H$. bacteriophora symbiotic or axenic nematodes and then measured the levels of ATP using a bioluminescence assay. We found that following nematode infection, there were no significant changes in ATP levels between either daw or dpp TGF- $\beta$ mutants compared to their background controls $\left(w^{1118}\right)$ (Figure 5). These results imply that the Activin and BMP TGF- $\beta$ signaling branches do not modulate ATP levels in $D$. melanogaster larvae during infection with $H$. bacteriophora nematodes.

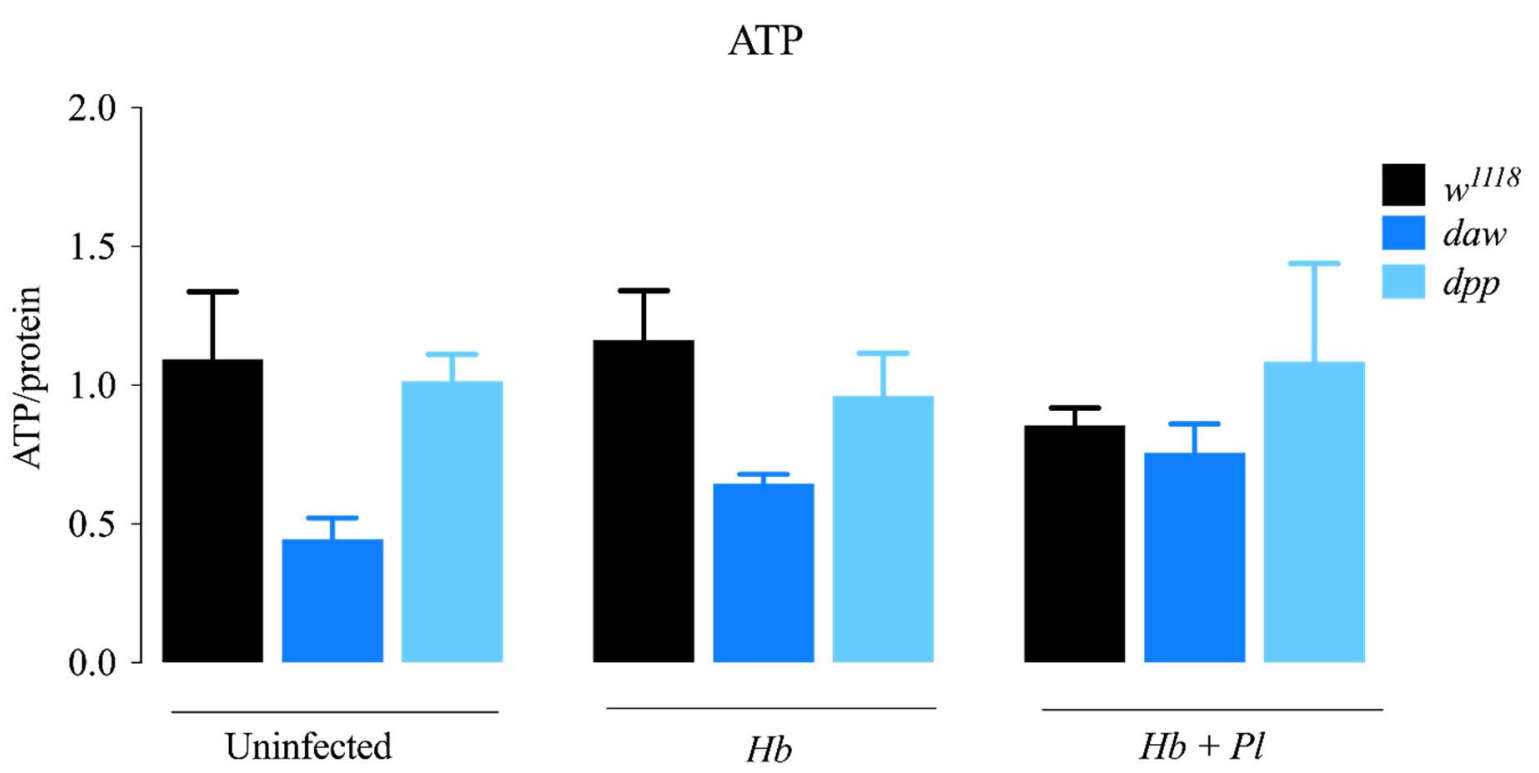

Figure 5. Levels of ATP in Drosophila melanogaster TGF-ß mutant larvae $24 \mathrm{~h}$ after infection with axenic (Heterorhabditis bacteriophora, $\mathrm{Hb}$ ) or symbiotic (Heterorhabditis bacteriophora + Photorhabdus luminescens, $\mathrm{Hb}+\mathrm{Pl}$ ) nematodes. There is no significant change in ATP levels between either daw or dpp TGF- $\mathrm{B}$ mutant and its background control $\left(w^{1118}\right)$. The experiment was performed in biological duplicates and repeated three times $(N=42-54)$.

\section{Discussion}

Pathogens and parasites improve and/or extend the duration of their infection capacity and transmissibility by inducing alterations in host metabolic processes [35,36]. Recent evidence has shown that the components of the Activin and the BMP branches of the TGF- $\beta$ signaling pathway contribute to the metabolic response of $D$. melanogaster larvae against infection with $H$. gerrardi nematodes, which naturally associate in a mutualistic symbiosis relationship with the bacteria P. asymbiotica [6]. However, whether the interaction between TGF- $B$ signaling activity and the D. melanogaster anti-nematode metabolic response is regulated by the nematodes alone without any potential influence from their natural found bacterial symbionts is not thoroughly understood. Therefore, we investigated the 
interaction between the Activin and the BMP signaling activity with the D. melanogaster metabolic response against infection with axenic or symbiotic $H$. bacteriophora nematodes. Here we report that the Activin signaling interacts with sugar metabolism while the BMP signaling mediates metabolism of lipids in D. melanogaster during the response to infection with axenic or symbiotic H. bacteriophora parasitic nematodes.

Previous studies have highlighted the importance of the Activin and BMP signaling branches in regulating $D$. melanogaster sugar metabolism in the absence of infection. The extracellular Activin branch ligand Daw regulates metabolic processes in D. melanogaster larvae in a positive manner. In addition, loss-of-function mutation in daw is associated with disruption in sugar homeostasis [30]. Similarly, loss-of-function mutation in the gene coding for the extracellular BMP branch ligand Gbb results in decreased glucose and trehalose levels [33]. In our study, we have also observed a link between Activin signaling activity and D. melanogaster sugar metabolism, however in the context of parasitic nematode infection. We have further demonstrated that daw deficient larvae contain elevated levels of trehalose in response to infection with axenic H. bacteriophora compared to their background control $\left(w^{1118}\right)$. In contrast, levels of trehalose were significantly decreased in daw mutants upon infection with symbiotic nematodes compared to uninfected daw mutants or larvae infected with axenic nematodes. This implies that the H. bacteriophora-P. luminescens nematode-bacteria complex can suppress the levels of trehalose in the absence of a functional Activin signaling branch. Of note, our previous results in D. melanogaster adults have revealed that injection of either P. luminescens or $P$. asymbiotica pathogenic bacteria into the hemolymph of wild type flies leads to a decrease in the levels of trehalose compared to uninfected individuals [37]. Taken together, our current findings suggest that the Activin branch of the TGF- $\beta$ signaling modulates trehalose metabolism in D. melanogaster larvae upon symbiotic $H$. bacteriophora nematode infection.

Lipid droplets consisting of TAGs and cholesteryl esters are considered as essential regulators of lipid metabolism [38]. Our previous studies have demonstrated that the change in lipid droplet status in the D. melanogaster larval fat body depends on the type of parasitic nematode infection, where Steinernema carpocapsae infection increases their size [39], but H. gerrardi infection results in a decrease [6]. In addition, no alterations in TAG levels were found in these studies. Similarly, in our current study we did not find any variation in TAG amounts and in addition no change in lipid droplet size was observed in the background control larvae responding to axenic or symbiotic H. bacteriophora infection compared to uninfected larvae. These differences might imply parasite-specific regulation of the host metabolic response. In addition, the Activin branch has a critical role in the regulation of lipid metabolism. The extracellular Activin signaling ligand Babo reduces TAG levels in the D. melanogaster larval fat body and promotes mobilization of lipids [40]. Our previous work has shown that the Activin and BMP branches participate in the regulation of lipid metabolism in D. melanogaster during response to parasitic nematode infection. More precisely, we have shown that lipid droplets in both $d a w$ and $d p p$ mutant larvae increase in size upon infection with $H$. gerrardi nematodes compared to uninfected larvae [6]. Here we report that lipid droplet size decreases in $d p p$ deficient larvae against infection with symbiotic $H$. bacteriophora nematodes compared to uninfected mutants. Both $H$. gerrardi (containing P. asymbiotica bacteria) and H. bacteriophora (containing P. luminescens bacteria) nematodes belong to the Heterorhabditidae family of parasitic nematodes, but they obviously interact differently with the host lipid droplets during infection. Hence, it is possible that variation in these effects might be due to differences in the biology of the symbiotic bacteria that inhabit the intestine of $H$. gerrardi and $H$. bacteriophora nematodes [41]. Interestingly, investigating the lipid content in BMP deficient larvae challenged with $H$. bacteriophora revealed a decrease in TAG levels in $d p p$ mutants upon symbiotic nematode infection and a decrease in cholesterol levels upon axenic nematode infection compared to their background controls. Our findings suggest that the BMP signaling branch modulates lipid metabolism through regulating TAG and cholesterol levels, which is reflected by changes in the size of fat body lipid droplets during $H$. bacteriophora infection. 
In summary, our study reports that the Activin signaling in D. melanogaster modulates sugar metabolism by increasing the levels of trehalose in response to symbiotic H. bacteriophora infection. In addition, the BMP signaling interacts with the metabolism of TAGs against infection with $H$. bacteriophora symbiotic nematodes, and the metabolism of cholesterol against infection with axenic nematodes. The BMP signaling also contributes to the regulation of fat body lipid droplets in larvae responding to $H$. bacteriophora nematode infection. Our observation that TGF- $\beta$ signaling activity in $D$. melanogaster can modify host metabolic processes during a response to infection with parasitic nematodes provides a new basis for the prevention and control of metabolic disorders associated with parasitic diseases in vertebrates, perhaps including humans. Knowledge of this information might contribute to the identification of alternative possibilities for adopting novel anti-parasitic treatment strategies in the future.

Author Contributions: Conceptualization, Y.O. and I.E.; methodology, Y.O.; software, Y.O.; formal analysis, Y.O.; investigation, Y.O., T.P., and D.R.; writing-original draft preparation, Y.O.; writing-review and editing, I.E.; visualization, Y.O.; supervision, I.E. and Y.O.; project administration, I.E. and Y.O.; funding acquisition, I.E. All authors have read and agreed to the published version of the manuscript.

Funding: This research was funded by the National Institute of Allergy and Infectious Diseases (grants 1R01AI110675-01A1 and 1R56AI110675-01).

Acknowledgments: We thank Kyle Devine for maintaining the D. melanogaster fly stocks, and members of the Department of Biological Sciences at George Washington University for critical reading of the manuscript. Also, we thank the Department of Biological Sciences at GW for providing summer Harlan research fellowships to Y.O. and D.R.

Conflicts of Interest: The authors declare no conflict of interest. The funders had no role in the design of the study; in the collection, analyses, or interpretation of data; in the writing of the manuscript, or in the decision to publish the results.

\section{References}

1. Melo, R.C.N.; Ávila, H.D.; Fabrino, D.L.; Almeida, P.E.; Bozza, P.T. Macrophage lipid body induction by Chagas disease in vivo: Putative intracellular domains for eicosanoid formation during infection. Tissue Cell 2003, 35, 59-67. [CrossRef]

2. Kapadia, S.B.; Chisari, F.V. Hepatitis C virus RNA replication is regulated by host geranylgeranylation and fatty acids. Proc. Natl. Acad. Sci. USA 2005, 102, 2561-2566. [CrossRef]

3. D'Avila, H.; Melo, R.C.N.; Parreira, G.G.; Werneck-Barroso, E.; Castro-Faria-Neto, H.C.; Bozza, P.T. Mycobacterium bovis Bacillus Calmette-Guérin Induces TLR2-Mediated Formation of Lipid Bodies: Intracellular Domains for Eicosanoid Synthesis In Vivo. J. Immunol. 2006, 176, 3087-3097. [CrossRef] [PubMed]

4. Anand, P.; Cermelli, S.; Li, Z.; Kassan, A.; Bosch, M.; Sigua, R.; Huang, L.; Ouellette, A.J.; Pol, A.; Welte, M.A.; et al. A novel role for lipid droplets in the organismal antibacterial response. Elife 2012, 1, e00003. [CrossRef] [PubMed]

5. Harsh, S.; Ozakman, Y.; Kitchen, S.M.; Paquin-Proulx, D.; Nixon, D.F.; Eleftherianos, I. Dicer-2 Regulates Resistance and Maintains Homeostasis against Zika Virus Infection in Drosophila. J. Immunol. 2018, 201, 3058-3072. [CrossRef] [PubMed]

6. Ozakman, Y.; Eleftherianos, I. TGF- $\beta$ signaling interferes with the Drosophila innate immune and metabolic response to parasitic nematode infection. Front. Physiol. 2019, 10, 716. [CrossRef]

7. Galenza, A.; Foley, E. Immunometabolism: Insights from the Drosophila model. Dev. Comp. Immunol. 2019, 94, 22-34. [CrossRef]

8. Stock, S.P.; Blair, H.G. Entomopathogenic nematodes and their bacterial symbionts: The inside out of a mutualistic association. Symbiosis 2008, 46, 65-75.

9. Castillo, J.C.; Reynolds, S.E.; Eleftherianos, I. Insect immune responses to nematode parasites. Trends Parasitol. 2011, 27, 537-547. [CrossRef]

10. Eleftherianos, I.; ffrench-Constant, R.H.; Clarke, D.J.; Dowling, A.J.; Reynolds, S.E. Dissecting the immune response to the entomopathogen Photorhabdus. Trends Microbiol. 2010, 18, 552-560. [CrossRef] 
11. Masucci, J.D.; Miltenberger, R.J.; Hoffmann, F.M. Pattern-specific expression of the Drosophila decapentaplegic gene in imaginal disks is regulated by 3' cis-regulatory elements. Genes Dev. 1990, 4, 2011-2023. [CrossRef] [PubMed]

12. Lecuit, T.; Brook, W.J.; Ng, M.; Calleja, M.; Sun, H.; Cohen, S.M. Two distinct mechanisms for long-range patterning by Decapentaplegic in the Drosophila wing. Nature 1996, 381, 387-393. [CrossRef] [PubMed]

13. Dobendsa, L.L.; Raftery, L.A. Drosophila Oogenesis: A Model System to Understand TGF- $\beta /$ Dpp Directed Cell Morphogenesis. Ann. N. Y. Acad. Sci. 1998, 857, 245-247.

14. Eleftherianos, I.; Castillo, J.C.; Patrnogic, J. TGF- $\beta$ signaling regulates resistance to parasitic nematode infection in Drosophila melanogaster. Immunobiology 2016, 221, 1362-1368. [CrossRef] [PubMed]

15. Patrnogic, J.; Heryanto, C.; Eleftherianos, I. Transcriptional up-regulation of the TGF- $\beta$ intracellular signaling transducer Mad of Drosophila larvae in response to parasitic nematode infection. Innate Immun. 2018, 24, 349-356. [CrossRef] [PubMed]

16. Raftery, L.A.; Sutherland, D.J. TGF- $\beta$ family signal transduction in Drosophila development: From Mad to Smads. Dev. Biol. 1999, 210, 251-268. [CrossRef]

17. Moustakas, A.; Heldin, C.H. Non-Smad TGF- $\beta$ signals. J. Cell Sci. 2005, 118, 3573-3584. [CrossRef]

18. Shi, Y.; Massagué, J. Mechanisms of TGF- $\beta$ signaling from cell membrane to the nucleus. Cell 2003, 113, 685-700. [CrossRef]

19. Peterson, A.J.; O'Connor, M.B. Strategies for exploring TGF- $\beta$ signaling in Drosophila. Methods 2014, 68, 183-193. [CrossRef]

20. Zi, Z.; Chapnick, D.A.; Liu, X. Dynamics of TGF- $\beta /$ Smad signaling. FEBS Lett. 2012, 2586, 1921-1928. [CrossRef]

21. White, G.F. A Method for Obtaining Infective Nematode Larvae From Cultures. Science (80-.) 1927, 66, 302-303. [CrossRef] [PubMed]

22. Kenney, E.; Hawdon, J.M.; O'Halloran, D.; Eleftherianos, I. Heterorhabditis bacteriophora Excreted-Secreted Products Enable Infection by Photorhabdus luminescens Through Suppression of the Imd Pathway. Front. Immunol. 2019, 10, 1-14. [CrossRef] [PubMed]

23. Tennessen, J.M.; Barry, W.E.; Cox, J.; Thummel, C.S. Methods for studying metabolism in Drosophila. Methods 2014, 68, 105-115. [CrossRef]

24. Mattila, J.; Hietakangas, V. Regulation of carbohydrate energy metabolism in Drosophila melanogaster. Genetics 2017, 207, 1231-1253. [CrossRef]

25. Nässel, D.R.; Liu, Y.; Luo, J. Insulin/IGF signaling and its regulation in Drosophila. Gen. Comp. Endocrinol. 2015, 221, 255-266. [CrossRef]

26. DiAngelo, J.R.; Birnbaum, M.J. Regulation of Fat Cell Mass by Insulin in Drosophila melanogaster. Mol. Cell. Biol. 2009, 29, 6341-6352. [CrossRef]

27. Géminard, C.; Arquier, N.; Layalle, S.; Bourouis, M.; Slaidina, M.; Delanoue, R.; Bjordal, M.; Ohanna, M.; Ma, M.; Colombani, J.; et al. Control of metabolism and growth through insulin-like peptides in Drosophila. Diabetes 2006, 55, 5-8. [CrossRef]

28. Grönke, S.; Clarke, D.F.; Broughton, S.; Andrews, T.D.; Partridge, L. Molecular evolution and functional characterization of Drosophila insulin-like peptides. PLoS Genet. 2010, 6, e1000857. [CrossRef]

29. Semaniuk, U.V.; Gospodaryov, D.V.; Feden'ko, K.M.; Yurkevych, I.S.; Vaiserman, A.M.; Storey, K.B.; Simpson, S.J.; Lushchak, O. Insulin-like peptides regulate feeding preference and metabolism in Drosophila. Front. Physiol. 2018, 9, 1-14. [CrossRef]

30. Ghosh, A.C.; O'Connor, M.B. Systemic Activin signaling independently regulates sugar homeostasis, cellular metabolism, and pH balance in Drosophila melanogaster. Proc. Natl. Acad. Sci. USA 2014, 111, 5729-5734. [CrossRef]

31. Liu, Z.; Huang, X. Lipid metabolism in Drosophila: Development and disease. Acta Biochim. Biophys. Sin. (Shanghai) 2013, 45, 44-50. [CrossRef] [PubMed]

32. Yang, L.; Roh, Y.S.; Song, J.; Zhang, B.; Liu, C.; Loomba, R.; Seki, E. Transforming growth factor beta signaling in hepatocytes participates in steatohepatitis through regulation of cell death and lipid metabolism in mice. Hepatology 2014, 59, 483-495. [CrossRef]

33. Ballard, S.L.; Jarolimova, J.; Wharton, K.A. Gbb/BMP signaling is required to maintain energy homeostasis in Drosophila. Dev. Biol. 2010, 337, 375-385. [CrossRef] 
34. Kühnlein, R.P. The contribution of the Drosophila model to lipid droplet research. Prog. Lipid Res. 2011, 50, 348-356. [CrossRef]

35. Freyberg, Z.; Harvill, E.T. Pathogen manipulation of host metabolism: A common strategy for immune evasion. PLoS Pathog. 2017, 13, e1006669. [CrossRef]

36. Shea-Donohue, T.; Qin, B.; Smith, A. Parasites, nutrition, immune responses and biology of metabolic tissues. Parasite Immunol. 2017, 39, e12422. [CrossRef]

37. Shokal, U.; Kopydlowski, H.; Harsh, S.; Eleftherianos, I. Thioester-Containing Proteins 2 and 4 Affect the Metabolic Activity and Inflammation Response in Drosophila. Infect. Immun. 2018, 86, 1-18. [CrossRef]

38. Jarc, E.; Petan, T. A twist of FATe: Lipid droplets and inflammatory lipid mediators. Biochimie 2020, 169, 69-87. [CrossRef]

39. Yadav, S.; Frazer, J.; Banga, A.; Pruitt, K.; Harsh, S.; Jaenike, J.; Eleftherianos, I. Endosymbiont-based immunity in Drosophila melanogaster against parasitic nematode infection. PLoS ONE 2018, 13, e0192183. [CrossRef]

40. Song, W.; Owusu-Ansah, E.; Hu, Y.; Cheng, D.; Ni, X.; Zirin, J.; Perrimon, N. Activin signaling mediates muscle-to-adipose communication in a mitochondria dysfunction-associated obesity model. Proc. Natl. Acad. Sci. USA 2017, 114, 8596-8601. [CrossRef] [PubMed]

41. Waterfield, N.R.; Ciche, T.; Clarke, D. Photorhabdus and a Host of Hosts. Annu. Rev. Microbiol. 2009, 63, 557-574. [CrossRef] [PubMed]

(C) 2020 by the authors. Licensee MDPI, Basel, Switzerland. This article is an open access article distributed under the terms and conditions of the Creative Commons Attribution (CC BY) license (http://creativecommons.org/licenses/by/4.0/). 